\title{
Host-guest and guest-guest interactions between xylene isomers confined in the MIL-47(V) pore system
}

\author{
An Ghysels, ${ }^{[\mathrm{a}]}$ Matthias Vandichel, ${ }^{[\mathrm{a}]}$ Toon Verstraelen, ${ }^{[\mathrm{a}]}$ Monique A. van der Veen, ${ }^{[\mathrm{b}]}$ \\ Dirk E. De Vos, ${ }^{[b]}$ Michel Waroquier, ${ }^{[a]}$ and Veronique Van Speybroeck ${ }^{[a]}$
}

[a] Center for Molecular Modeling, Quantum Chemistry-Molecular Modeling Alliance, Ghent University, Technologiepark 903, 9052 Gent, Belgium

[b] Centre for Surface Chemistry and Catalysis, KU Leuven, Kasteelpark Arenberg 23, 3001 Heverlee, Belgium

\begin{abstract}
The porous MIL-47 material shows a selective adsorption behavior for para, ortho, and meta-isomers of xylenes, making the material a serious candidate for separation applications. The origin of the selectivity lies in the differences in interactions (energetic) and confining (entropic). This paper investigates the xylene-framework interactions and the xylene-xylene interactions with quantum mechanical calculations, using a dispersion corrected density functional and periodic boundary conditions to describe the crystal. First, the strength and geometrical characteristics of the optimal xylene-xylene interactions are quantified by studying the pure and mixed pairs in gas phase. An extended set of initial structures is created and optimized to sample as many relative orientations and distances as possible. Next, the pairs are brought in the pores of MIL-47. The interaction with the terephthalic linkers and other xylenes, increases the stacking energy in gas phase $(-31.7 \mathrm{~kJ} / \mathrm{mol}$ per pair) by roughly a factor four in the fully-loaded state $(-58.3 \mathrm{~kJ} / \mathrm{mol}$ per xylene). Our decomposition of the adsorption energy shows various trends in the contributing xylene-xylene interactions. The absence of a significant difference in energetics between the isomers indicates that entropic effects must be mainly responsible for the separation behavior.
\end{abstract}

Corresponding authors: an.ghysels@ugent.be, veronique.vanspeybroeck@ugent.be 


\section{Introduction}

Since a couple of years metal organic frameworks (MOFs) are a topical theme; they represent a new class of porous materials with extraordinary physicochemical and mechanical properties due to their hybrid architecture of organic and inorganic building blocks [1-3]. Their applications in the field of gas adsorption, storage, gas separation, catalysis are almost inexhaustible due to the specificity of the hybrid organic-inorganic composition [4-7]. Examples of their fascinating properties are the flexibility of the framework, the appearance of catalytic sites in the pores, etc. These are a consequence of the unique combination of properties typical for metals and organic species.

In previous work, some of the authors showed that Metal-Organic Frameworks could be successfully used as selective adsorbents for the industrially relevant separations of paraxylene $(\mathrm{pX})$ versus meta-xylene $(\mathrm{mX})$ and ortho-xylene $(\mathrm{oX})$ versus ethylbenzene $(\mathrm{eB})$ [8-10]. The separation of mixed C8 alkylaromatic compounds is one of the most challenging issues in the chemical industry, for example because of its direct link with PET production [11]. Among the various types of MOFs that have been tested, the MIL-47 material proved to be very successful. This MOF consists of one-dimensional pores in which terephthalate ligands are connected by $\mathrm{VO}^{2+}$ ions (Figure 1). MIL-47 is one of an impressive series of MOFs that were synthesized by Férey and co-workers [12]. It is not completely clear what mechanism is responsible for the preference of adsorption between the three types of xylene isomers and ethylbenzene. In this paper we try to unravel the molecular interactions between pairs of adsorbed xylenes and between xylenes and organic linkers using in silico experiments. The interactions are studied with quantum mechanical periodic modeling. Once the mechanism of selectivity in adsorption has been understood, the obtained knowledge can be used for designing new materials with tailor-made properties towards gas separation and to possibly modify the organic linkers to control the affinity for specific aromatic adsorbates. 


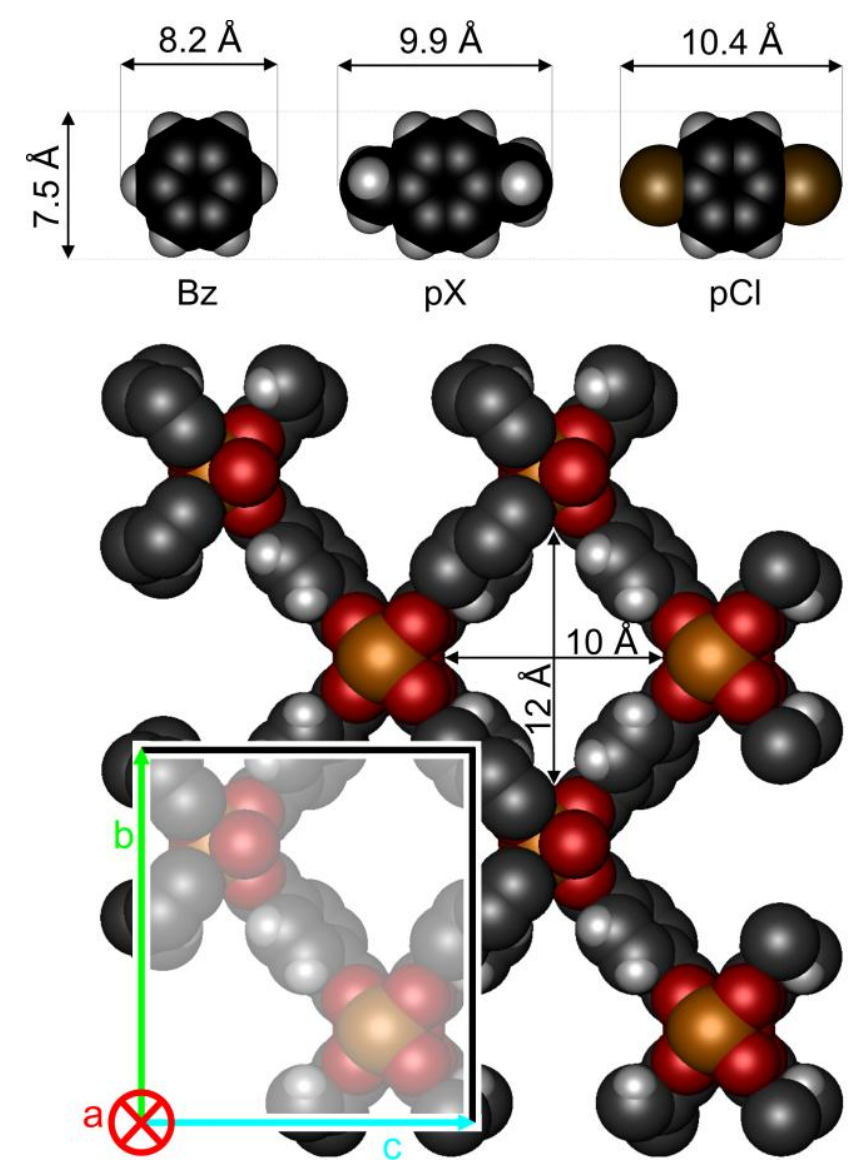

Figure 1 MIL-47 consists of terephthalic linkers connected by $\mathrm{VO}^{2+}$ ions. Xylenes adsorb well in the onedimensional pore channels. View along the $a$ direction; four unit cells are displayed. The pore dimensions of MIL-47 and the characteristic dimensions of the adsorbates are estimated by assigning van der Waals radii to the atoms.

Long range dispersion interactions and more particularly $\pi-\pi$ stacking interactions will manifestly play an important role for the organization of xylenes in the pore. Basically, only very advanced $a b$ initio correlated wavefunction methods are able to account for these effects, but they are computationally too demanding to apply to our system. Nowadays, Density Functional Theory (DFT) is the most widely used method for electronic structure calculations for extended molecular systems, but without modifications these methods are not able to account for long-range electrostatic correlations that are responsible for the dispersion forces. Grimme and co-workers proposed the DFT-D method in which an empirical $C_{6} R^{-6}$ correction is added to the standard density functionals [13-15]. This method is able to account for the $\pi-\pi$ interactions in MOFs [14].

Several molecular modeling studies investigated various adsorbates with grand canonical Monte Carlo techniques (GCMC) on MIL-47: $\mathrm{N}_{2}$ [16], $\mathrm{CO}_{2}$ [17], $\mathrm{CH}_{4}$ [18], $\mathrm{H}_{2}$ [19], xylenes [20]. GCMC techniques usually rely on fast classical force field descriptions of the global potential energy of the system. So, GCMC techniques allow a fast computation of 
thermodynamic quantities and they have been frequently and successfully applied to explain separations over MOFs [16, 20-24]. The force fields required to perform the molecular simulations are taken from literature. The accuracy of these classical approaches will certainly be improved in the near future when more advanced force fields are available for these particular MOFs that also account for the flexibility of the framework [25]. In the MIL-47 study by Castillo et al. [20], good agreement is found with isotherm experiments using a model with partial atomic charges and the Lennard-Jones potential for the van der Waals interactions. The framework is kept rigid during the simulations. The computed mixture isotherms of the xylene isomers lead to separation factors in MIL-47 predicting a preferential adsorption for ortho-xylene (oX), closely followed by para (pX), and then meta (mX). The adsorption selectivity was found to increase with pressure and the results agree with the experimental findings. The computed adsorption isotherms manifestly overestimate the loading of para and ortho-xylene at the lowest temperatures. The authors attribute this discrepancy to small changes of the framework structure with temperature. The saturation loading reached in their simulations was about 4 molecules per unit cell while experimentally the maximum loading was 3.4 molecules per unit cell $[8,9]$.

The selectivity of xylene isomers in MIL-47 most likely originates from packing effects, as suggested by Alaerts and Finsy et al. [8, 9], It has been speculated that molecules are adsorbed by pairs at high loadings, their benzene rings facing each other and approximately parallel to the aromatic rings of the teraphthalate linkers. Additionally, the spatial arrangement of the methyl groups of each xylene should play a significant role in the determination of the preferential adsorption. It is suspected that the crude force field used in the work of Castillo et al. cannot properly describe these packing effects. With the current lack of suitable more accurate force fields, quantum chemical DFT-D calculations can give some added value in the underlying mechanism for the selective adsorption of xylene isomers. Stacking effects are more accurately described when taking into account the long-range dispersion interactions by using the empirical Grimme corrections [13] complementary to the standard density functionals. A DFT-D approach has also the advantage that the adsorption and stacking energy can be decoupled in various contributions elucidating the interactions responsible for the observed different adsorption behavior. Unlike the molecular simulation of Castillo et al. [20], we also incorporate in this study the packing of mixed xylene pairs such as $\mathrm{pX}-\mathrm{oX}, \mathrm{pX}$ $\mathrm{mX}$ and $\mathrm{oX}-\mathrm{mX}$. 
In this work, we first investigate xylene-xylene interactions in the gas phase. The strength of the xylene stacking energy is compared to the stacking energy of benzene pairs and dichlorobenzene pairs. Benzene stacking is well studied [26-28], and dichlorobenzene adsorption shows experimentally a similar selectivity behavior in MIL-47 as xylenes [29]. Next, the pairs are embedded in the MIL-47 framework respecting the maximum loading of two xylene pairs in one unit cell. An extensive set of configurations for the adsorbed xylenes is generated. As such, we are able to validate the effect of the confined environment and interaction with the walls of the material. The adsorption energy of the most stable configuration is studied in detail using an energy decomposition scheme to achieve more insight into the host-guest and guest-guest interactions. 


\section{Computational methods}

\subsection{Characterization of a xylene pair}

A thorough study on the interaction between xylene pairs will be performed, and thus it is essential to give a good definition of their relative position with respect to each other. The relative position and orientation of a xylene pair is characterized by attaching a Cartesian coordinate frame to each xylene (Figure 2a) [30] denoted as $\left(O_{1}, \boldsymbol{x}_{1}, \boldsymbol{y}_{1}, \boldsymbol{z}_{1}\right)$ and $\left(O_{2}, \boldsymbol{x}_{2}, \boldsymbol{y}_{2}, \boldsymbol{z}_{2}\right)$ for the two xylenes, $\mathrm{X}_{1}$ and $\mathrm{X}_{2}$, respectively. The $\boldsymbol{x}$-axis always coincides with the twofold rotational symmetry axis of each xylene. Note that in the three-dimensional structure, the orientation of the methyl groups may prevent the existence of this $C_{2}$ symmetry, but the symmetry axis is always present in the projection on paper (Figure 2a). The sense of $z_{2}$ is choosen such that the angle between $z_{1}$ and $z_{2}$ is less than $90^{\circ}$. In principle, the relative orientation of two molecules is completely characterized by six parameters. For this work we only consider the four most important internal coordinate parameters to describe their relative orientation: distance (d), shift (s), tilt $(\theta)$, and phase $(\varphi)$. These are defined below such that an interchange of $X_{1}$ and $X_{2}$ or a point inversion results in the same values for these four parameters.

The distance parameter $d$ is the length of the projection of the vector $\boldsymbol{R}$ (connecting the centers of the six-membered rings) on the vector $z_{1}+z_{2}$ (Figure $2 b$ ). The length of the orthogonal complement in this projection is called the shift $s$. Next, $\mathrm{X}_{2}$ is translated over the vector $\boldsymbol{R}$ to bring the geometric centers of the six-membered rings on top of each other (Figure $2 \mathrm{c}$ ). The tilt $\theta$ is defined as the angle between the $z_{1^{-}}$and $z_{2}$-axis. $X_{2}$ is then rotated over $\theta$ about the vector $z_{1} \times z_{2}$, such that $\left(O_{2}, x_{2}, y_{2}, z_{2}\right) \rightarrow\left(O_{2}, x_{2}, y_{2}, z_{1}\right)$; the $z$-axes of the xylenes are now aligned and the $\boldsymbol{x}$-axes and $\boldsymbol{y}$-axes lie in the same plane (Figure 2d). Finally, the phase $\varphi$ is determined as the absolute value of the angle between the $\boldsymbol{x}_{\boldsymbol{1}^{-}}$and $\boldsymbol{x}_{\mathbf{2}}$ '-axis.

The above procedure is also applied to a benzene pair and dichlorobenzene pairs. 


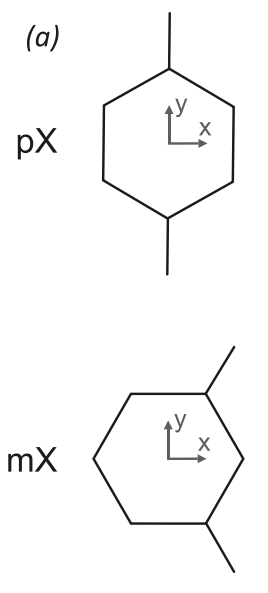

(b)

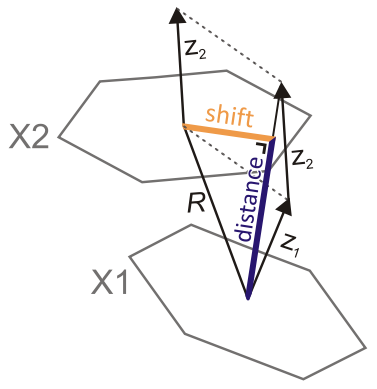

(c)

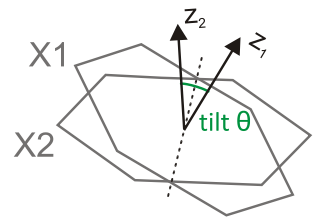

oX<smiles>Cc1ccccc1C</smiles>

(d)

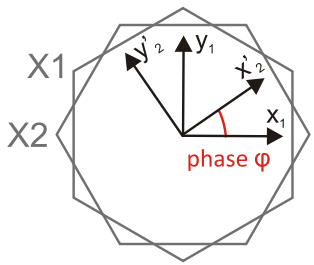

Figure 2 A coordinate system is attached to each xylene $\left(X_{1}\right.$ and $\left.X_{2}\right)$ in a xylene pair. Four parameters are defined to describe their relative position and orientation: shift $(s)$, distance $(d)$, tilt $(\theta)$, and phase $(\varphi)$. 


\subsection{Quantumchemical modeling}

Density functional theory calculations are carried out using the CPMD code which allows to simulate systems with periodic boundary conditions[31]. The BP exchange correlation functional [32] is used with ultrasoft Vanderbilt pseudopotentials [33], especially designed to work with a 25 Ry cut off for the auxiliary plane wave grid. To include the possible effects of $\pi-\pi$ interactions, the BP-functional is combined with the Grimme dispersion correction for van der Waals interactions [13]. Three types of systems are simulated: xylenes in vacuum, the empty MIL-47 framework, and the fully loaded MIL-47 framework with four xylenes per unit cell.

Vacuum calculations of a xylene molecule or a pair of xylenes are performed in a large box of $20 \times 20 \times 20 \AA^{3}$ and are labeled with 'vac'. The investigated molecules are para-xylene (pX), ortho-xylene (oX) and meta-xylene (mX). In addition, benzene (Bz) and dichlorobenzenes in para, ortho, and meta configuration $(\mathrm{pCl}, \mathrm{oCl}, \mathrm{mCl})$ are simulated in vacuum for comparison.

The initial framework geometry of the empty MIL-47 is taken directly from the refined structure obtained by X-ray diffraction (Supp. Info. of Ref. [12], CCDC-166785, orthorhombic unit cell with $a=6.8179 \AA, b=16.1433 \AA, c=13.9392 \AA)$. In the energy minimization, the internal coordinates are optimized while the unit cell parametes are kept fixed. The metal $\mathrm{V}^{4+}$ ions in MIL-47 have each spin $1 / 2$. The unit cell containing four vanadium centers can have a total spin of 0,1 or 2 . The most stable structure corresponds with $S=1$ and the gain in energy with respect to the $S=0$ system amounts to $80 \mathrm{~kJ} / \mathrm{mol}$ per unit cell. As adsorption energies require the computation of differences in energy, the differences due to the usage of another spin state cancel out if we consistently use the same total spin for the framework. In view of the computational cost, all calculations were performed with $S=0$.

In the fully loaded state, two xylene pairs are inserted in the pores of MIL-47 with the Zeobuilder package [34] and the geometry is optimized with CPMD [31]. The experimental unit cell parameters are used when MIL-47 is fully loaded with pure $\mathrm{pX}$, oX, or $\mathrm{mX}$ (codes CCDC-632101, CCDC-632102, CCDC-632103, respectively [8]). Because of lack of experimental values during multi-component adsorption experiments, the empty framework parameters are used when MIL-47 is fully loaded with a mixture of the xylene isomers (code CCDC-166785 [12]). The decomposition of adsorption energies in Section 3 is derived from static calculations, where xylenes or framework atoms are removed from the fully loaded state 
and the energy is calculated without prior geometry optimization. These energies are labeled with 'stat'. 


\section{Results and discussion}

\subsection{Guest-guest interactions in gas phase}

Before tackling the xylenes interactions in the MIL-47 pore system, it is useful to have an understanding of the stacking of xylenes in the gas phase. Therefore, we construct a series of xylene pairs, and the geometry and the stacking energy is investigated for each of them. The geometry is characterized by the four geometry parameters in Figure 2: shift, distance, tilt, and phase. The stacking energy is calculated from the optimized geometry of the individual xylene molecules $\mathrm{X}_{1}$ and $\mathrm{X}_{2}$ and of a xylene pair $\mathrm{P}_{12}$. The stacking energy is defined as

$$
\Delta E_{\text {stack }}^{\text {vac }}\left(P_{12}\right)=E^{v a c}\left(P_{12}\right)-E^{v a c}\left(X_{1}\right)-E^{v a c}\left(X_{2}\right)
$$

Six pairs are studied: 'pure' pairs (pX-pX, oX-oX, mX-mX) and 'mixed' pairs (oX-mX, oX-pX, $\mathrm{pX}-\mathrm{mX}$ ). An extensive set of initial structures (Figure 3) is generated by varying the distance $d$ and the phase $\varphi$ between the pairs: $d$ varies between $2.8 \AA$ and $4.6 \AA$ with steps of $0.2 \AA$, while $\varphi$ is increased with steps of $30^{\circ}$ until a symmetrically equivalent structure is met. The initial shift $s$ and tilt $\theta$ are set to zero. The geometries of these 330 initial structures are optimized in a large box (vacuum). For each pair, the optimized geometries are ordered according to increasing energy $\left(E_{1}, E_{2}, \ldots\right)$. 


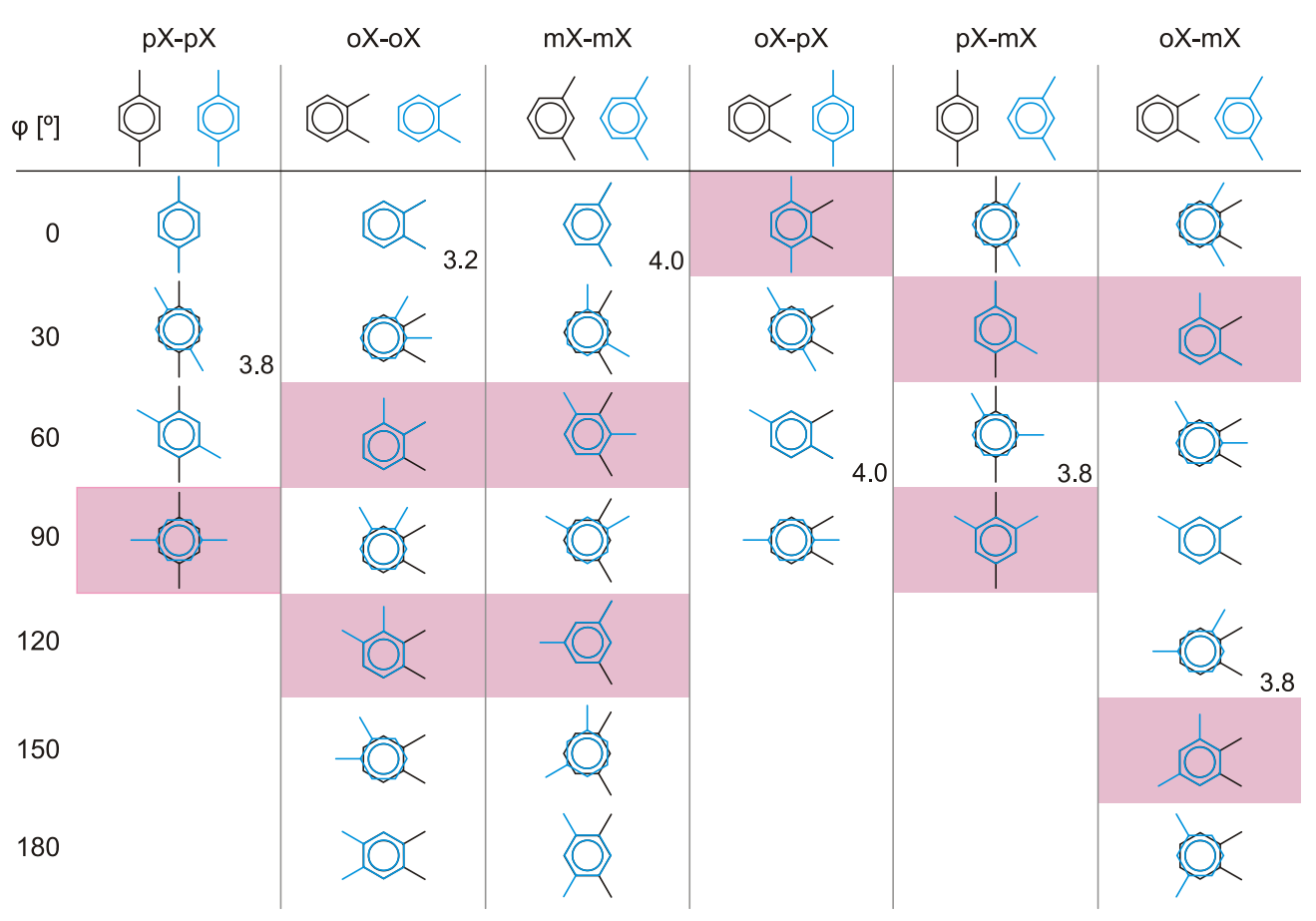

Figure 3 Initial geometries of six xylene pairs are obtained by varying the phase $\varphi$ and the distance $d$. A similar study is performed for dichlorobenzene pairs. Orientations that do not fit in the MIL-47 pores are given a red background. The initial geometries leading to the most stable structure are labeled with the initial distance.

Table 1 presents the geometrical properties and stacking energies for the following two cases. First, the geometrical properties are calculated for the geometry with the lowest energy $E_{1}$. Second, in case of thermal equilibrium, the thermal average of a property $A$ is obtained by weighting the contribution of each geometry by its Boltzmann probability $p\left(E_{i}\right)$,

$$
\begin{gathered}
p\left(E_{i}\right)=\exp \left(-\frac{E_{i}}{k_{B} T}\right) / \sum_{j} \exp \left(-\frac{E_{j}}{k_{B} T}\right) \\
<A>=\sum_{i} p\left(E_{i}\right) A(i)
\end{gathered}
$$

with $T$ the temperature and $k_{B}$ the Boltzmann constant. Due to temperature, not only the lowest energy state $E_{l}$ of a pair is populated, but also nearby minima can be reached. Note however that our formula only sums over local minima but no nearby non-equilibrium states, which would be required for accurate thermodynamics. 


\begin{tabular}{|c|c|c|c|c|c|c|c|c|c|c|c|}
\hline & \multicolumn{5}{|c|}{ Lowest energy geometry } & \multirow{2}{*}{$\frac{\mathbf{3 0 0} \mathbf{K}}{p\left(E_{1}\right)}$} & \multicolumn{5}{|c|}{ Thermal average $300 \mathrm{~K}$} \\
\hline & $\begin{array}{c}\text { shift } \\
(\AA)\end{array}$ & $\begin{array}{c}\text { distance } \\
(\AA)\end{array}$ & $\begin{array}{l}\text { tilt } \\
\left({ }^{\circ}\right)\end{array}$ & $\begin{array}{c}\text { phase } \\
\left({ }^{\circ}\right)\end{array}$ & $\begin{array}{c}\Delta E_{\text {stack }}^{\text {vac }} \\
(\mathrm{kJ} / \mathrm{mol})\end{array}$ & & $\begin{array}{c}\text { shift }> \\
(\AA)\end{array}$ & $\begin{array}{c}\text { distance }> \\
(\AA)\end{array}$ & $\begin{array}{c}\text { tilt }> \\
\left({ }^{\circ}\right)\end{array}$ & $\begin{array}{c}\text { phase }> \\
\left({ }^{\circ}\right)\end{array}$ & $\begin{array}{c}\left\langle\Delta E_{\text {stack }}^{\text {vac }}\right\rangle \\
(\mathrm{kJ} / \mathrm{mol})\end{array}$ \\
\hline $\mathrm{Bz}-\mathrm{Bz}$ & 1.66 & 3.22 & 5.5 & 9.0 & -19.7 & 1.0 & 1.66 & 3.22 & 5.5 & 9.0 & -19.7 \\
\hline $\mathrm{pX}-\mathrm{pX}$ & 0.8 & 3.27 & 3.3 & 38.5 & -31.1 & 0.3 & 0.6 & 3.31 & 4.0 & 80.3 & -28.5 \\
\hline oX-oX & 1.3 & 3.25 & 2.6 & 41.9 & -32.6 & 0.8 & 1.1 & 3.30 & 3.3 & 47.6 & -30.3 \\
\hline$m X-m X$ & 1.5 & 3.22 & 4.7 & 104.9 & -33.0 & 0.4 & 1.2 & 3.28 & 5.3 & 75.1 & -30.6 \\
\hline $\mathrm{pX}-\mathrm{oX}$ & 1.3 & 3.34 & 4.2 & 102.5 & -34.5 & 0.3 & 1.3 & 3.30 & 3.6 & 162.7 & -32.7 \\
\hline $\mathrm{pX}-\mathrm{mX}$ & 1.4 & 3.20 & 8.9 & -15.8 & -36.3 & 0.5 & 1.3 & 3.23 & 7.5 & 29.0 & -33.8 \\
\hline $\mathrm{oX}-\mathrm{mX}$ & 1.5 & 3.28 & 1.0 & -26.9 & -37.1 & 0.5 & 1.3 & 3.27 & 2.4 & -25.3 & -34.4 \\
\hline Average & 1.3 & 3.26 & 4.1 & 40.8 & -34.1 & 0.4 & 1.1 & 3.28 & 4.4 & 61.6 & -31.7 \\
\hline $\mathrm{pCl}-\mathrm{pCl}$ & 1.1 & 3.17 & 4.1 & 59.4 & -28.0 & 0.3 & 0.5 & 3.30 & 3.2 & 80.5 & -24.1 \\
\hline $\mathrm{oCl}-\mathrm{oCl}$ & 0.9 & 3.27 & 2.9 & 124.6 & -27.8 & 0.2 & 0.6 & 3.33 & 2.4 & 140.5 & -25.2 \\
\hline $\mathrm{mCl}-\mathrm{mCl}$ & 0.4 & 3.40 & 9.6 & 64.0 & -24.8 & 0.1 & 0.3 & 3.40 & 5.0 & 85.8 & -21.6 \\
\hline $\mathrm{pCl}-\mathrm{oCl}$ & 0.9 & 3.28 & 1.9 & 152.5 & -25.1 & 0.1 & 0.5 & 3.36 & 4.9 & 168.8 & -22.5 \\
\hline $\mathrm{pCl}-\mathrm{mCl}$ & 0.9 & 3.23 & 5.9 & 91.7 & -28.3 & 0.2 & 0.8 & 3.29 & 4.2 & 84.5 & -25.3 \\
\hline $\mathrm{oCl}-\mathrm{mCl}$ & 1.0 & 3.24 & 2.5 & -149.5 & -29.9 & 0.3 & 0.8 & 3.29 & 2.3 & -123.1 & -27.0 \\
\hline Average & 0.9 & 3.27 & 4.5 & 57.1 & -27.3 & 0.2 & 0.6 & 3.33 & 3.7 & 72.8 & -24.3 \\
\hline
\end{tabular}

Table 1 The geometry parameters (shift $s$, distance $d$, tilt $\theta$, and phase $\varphi$ ) and stacking energy (Eq. (1)) calculated for the optimized structure with lowest energy and calculated as a thermal average. The probability $p\left(E_{1}\right)$ to find the structure in the lowest energy state at $300 \mathrm{~K}$ is also listed. Averages in the table are taken over the six xylene pairs and over the six dichlorobenzene pairs.

The probability distribution $p\left(E_{i}\right)$ favors the lowest energy geometry $E_{1}$ largely at $300 \mathrm{~K}$ (highly peaked distribution in Supp. Info. Fig. 1), making this geometry the dominant contribution to the sum in Eq. (3). Indeed, the lowest energy properties correlate well with the thermal average properties in Table 1. Figure 4 illustrates the shift and distance parameters in the optimized benzene pair and the most stable pure xylene pairs. Clearly, the pairs take the parallel-displaced configuration. 

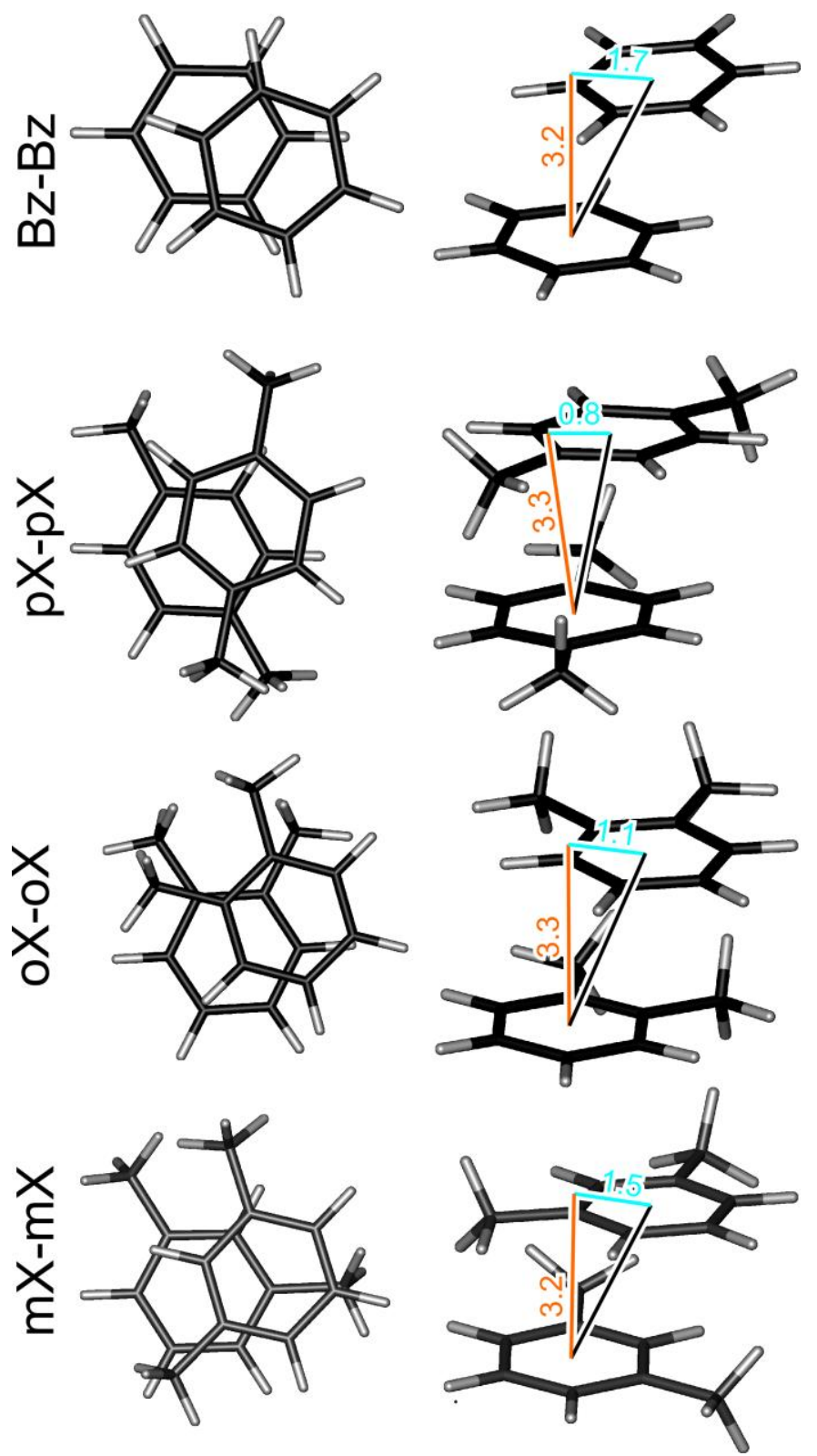

Figure 4 Some stacked pairs in the gas phase viewed along the $z_{1}$ axis and sideways: benzene pair and pure $\mathrm{pX}, \mathrm{oX}$, and $\mathrm{mX}$ xylene pairs. The orange and blue line indicate the distance $d$ and shift $s$ parameters, respectively.

The stacking energy $\Delta E_{\text {stack }}^{v a c}$ is favorable for benzene, xylenes, and dichlorobenzenes. Xylene pairs are the most stable with an average stacking energy of $-31.7 \mathrm{~kJ} / \mathrm{mol}$, followed by the dichlorobenzene pairs with $-24.3 \mathrm{~kJ} / \mathrm{mol}$ and benzene pairs with $-19.7 \mathrm{~kJ} / \mathrm{mol}$. Aromatic $\pi$ $\pi$ stacking has been extensively studied for benzene pairs [35-43], and it was found that Tshaped and parallel-displaced configurations have nearly equal stacking energy, being slightly more stable than the face-to-face sandwich configuration [26-28]. The benzene pair geometry in Table 1 is the parallel-displaced configuration. These studies also indicate that substituents, typically make the stacking stronger with respect to benzene stacking, which is confirmed in our results for methyl and chlorine substituents [44-46]. 
Moreover, Table 1 suggests a relationship between the stacking energy and some of the geometrical parameters. The absolute value of the stacking energy correlates negatively with the distance (Figure 5) and positively with the shift, whereas it appears to be relatively independent of the tilt and the phase. This means that the most favorable stacking is obtained when the rings lie close to each other and are somewhat shifted. Indeed, perfectly stacked rings with zero shift are less stable than parallel-displaced rings. The xylenes have higher shifts and lower distances than the dichlorobenzenes, thus explaining the systematically stronger stacking energy of the xylenes. The benzene pair is an outlier and does not follow this trend.
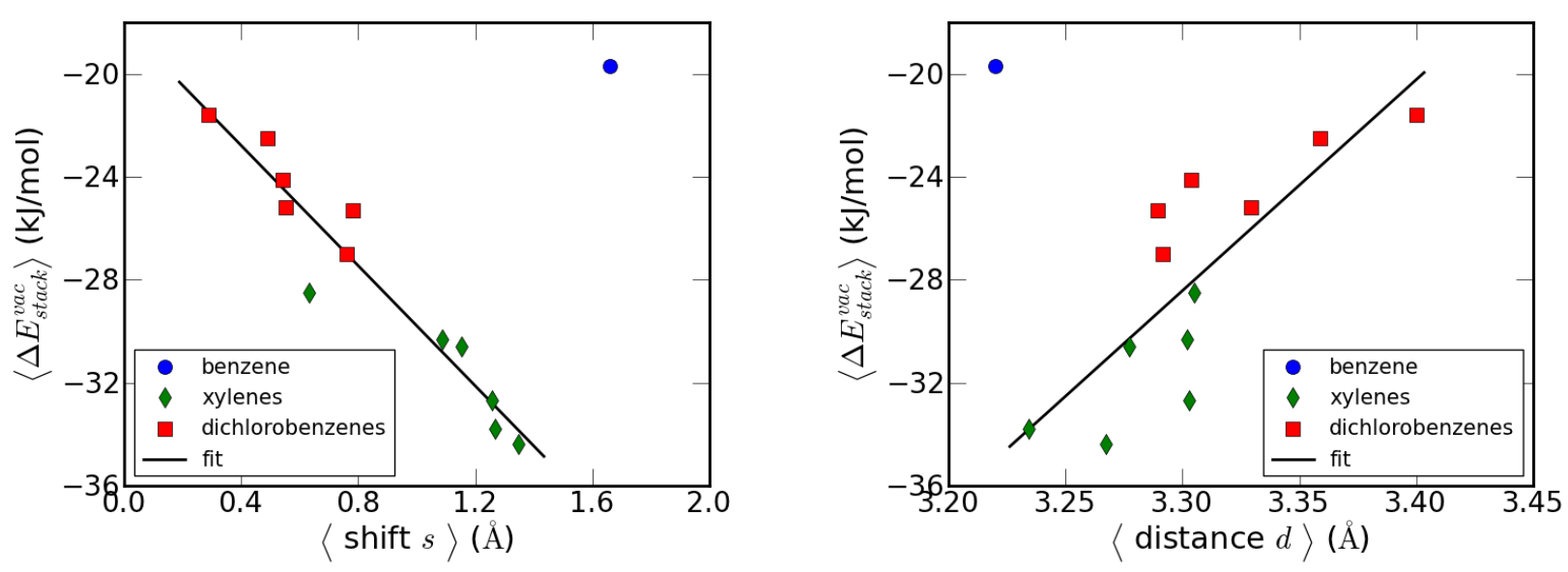

Figure 5 The stacking energy of the pairs becomes stronger with increasing shift $s$ and decreasing distance $d$. The benzene pair is an outlier; the linear fits are based on the thermal average values at $300 \mathrm{~K}$ of the xylene and dichlorobenzene pairs.

\subsection{MIL-47 packed with four xylenes: decomposition of the adsorption energy}

The stacking of xylenes is a favorable interaction in the gas phase of the order of -31.7 $\mathrm{kJ} / \mathrm{mol}$. Since the walls of the MIL-47 pores contain aromatic rings in the terephthalic linkers, it is expected that adsorbed xylenes are stabilized by an additional stacking energy: the interaction between the adsorbed xylenes and the framework, i.e. the so-called host-guest interactions. We have investigated the geometry characteristics and adsorption energy when MIL-47 is loaded with a xylene pair in each of its pores, which amounts to a total of four xylenes (two pairs) per unit cell. The adsorption energy is calculated as the difference in 
energy between the framework wherein two pairs $\left(P_{12}, P_{34}\right)$ are adsorbed, the empty framework $(F)$, and the individual xylene molecules $\left(X_{1}, X_{2}, X_{3}, X_{4}\right)$,

$$
\begin{gathered}
\Delta E_{a d s}\left(P_{12}, P_{34}\right)=E\left(F, P_{12}, P_{34}\right)-E(F)-E^{v a c}\left(X_{1}\right)-E^{v a c}\left(X_{2}\right) \\
-E^{v a c}\left(X_{3}\right)-E^{v a c}\left(X_{4}\right)
\end{gathered}
$$

Both guest-guest and guest-host interactions contribute to this adsorption energy.

In order to find the most favorable configurations of xylene pairs in the framework, an extensive set of initial structures is generated in a similar fashion as in the gas phase analysis in Section 2.1. Each unit cell contains two pores, and each pore is filled with a xylene pair. As in the gas phase, the list of pair geometries is generated systematically by varying the distance $d$ between $2.8 \AA$ and $4.0 \AA$ in steps of $0.2 \AA$ and by varying the phase $\varphi$ in steps of $30^{\circ}$. This gives 231 initial pair geometries, as shown in Figure 3. However, the confinement in the pore prevents some of the proposed pairs to be adsorbed (these orientations are given a red background in Figure 3), leaving 161 plausible initial pair geometries. We adopt now the following procedure, as depicted in Figure 6: for each of the plausible pair geometries, a duplicate is placed in the center of the first pore and another in the center of the second pore. This procedure results in 161 initial structures for fully loaded MIL-47.

An extra degree of freedom in the adsorbed state, compared to the gas phase, is the relative orientation of a pair as a whole with respect to the framework. Whereas rotating a pair or applying symmetry operations does not affect the energy in vacuum, this symmetry is broken when a pair is brought in the pores. Our 161 initial structures only represent 23 possible pair orientations. Unfortunately, a full sampling of this orientational degree of freedom is computationally not feasible. Nevertheless, we have added 15 orientations to improve the sampling, mainly for the mixed pairs which have lower symmetry than the pure pairs. In total, 266 initial structures for the fully loaded MIL-47 are created and optimized. 

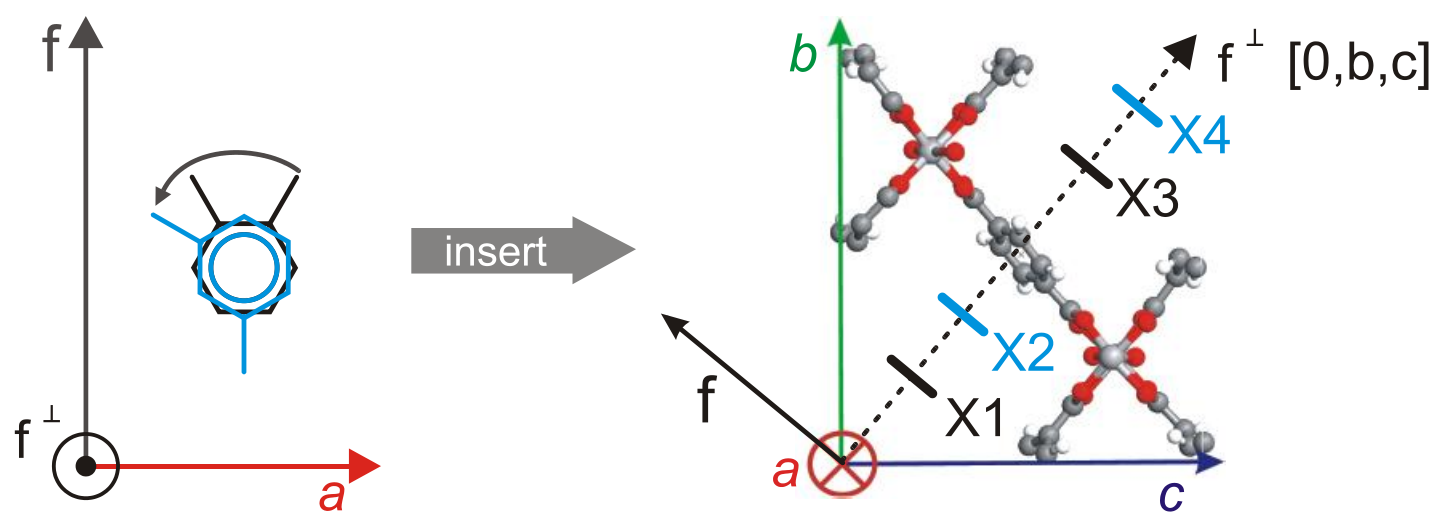

Figure 6 The initial geometries of the fully loaded framework are created by inserting the xylene pairs of Figure 3 into the MIL-47 pores. The vectors $f$ and $f^{\perp}$ denote the diagonals in the $(b, c)$ plane, orthogonal to the $a$ direction. The pairs are inserted at the origin and translated over the vector $0.5 a$. Subsequently, one pair is translated over the vector $0.25 f \perp$ and the other over the vector $0.75 f \perp$.

The geometry of each initial structure for the fully loaded state is optimized. Figure 7 shows the resulting geometries of the adsorbed pure pairs, and the mixed pairs are given in Figures 6-8 of the Supp. Info. For a given pore filling, the geometries are ordered according to increasing energy and the probability distribution is calculated (Eq. (2)). Since the Boltzmann distribution is peaked (Supp. Info. Fig. 2), only the dominant geometry with the lowest energy is considered in the remainder of this section.

$\mathrm{pX}$
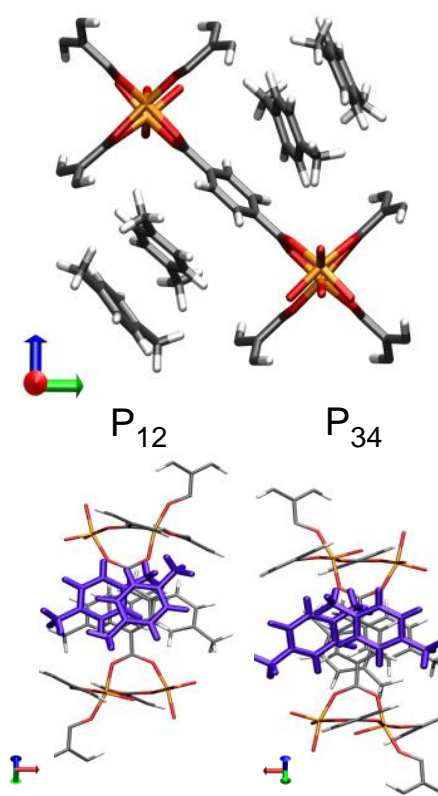

$\mathrm{P}_{34}$

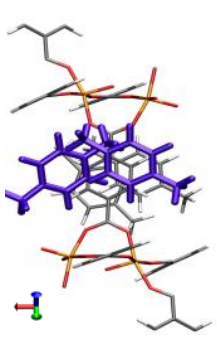

oX

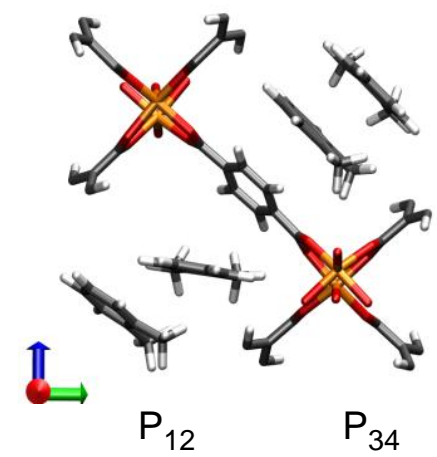

F

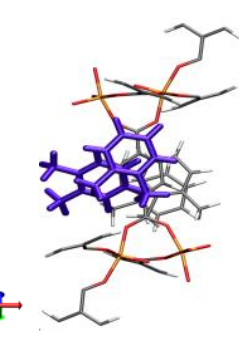

$\mathrm{mX}$

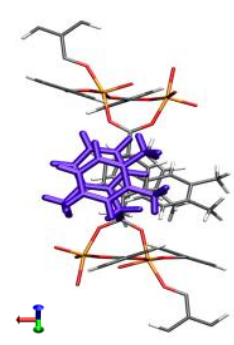

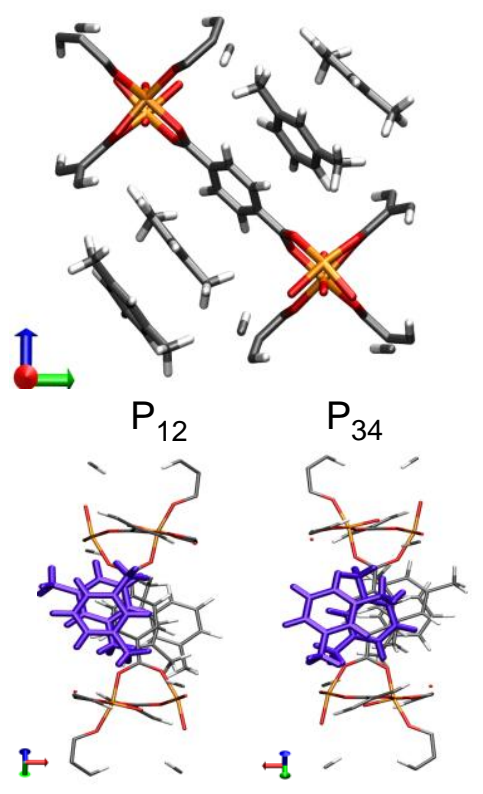

Figure 7 The most stable geometry of the pure $\mathbf{p X}, \mathbf{o X}$, and $\mathbf{m X}$ pairs adsorbed in MIL-47. Top: one unit cell viewed along the one-dimensional channels, in the $a$ direction (red arrow), loaded with four xylenes. Bottom: for each adsorbed state, the geometry of the $P_{12}$ and $P_{34}$ pairs is highlighted in purple in a view along the vector $f^{\perp}$, as defined in Figure 6 , for $P_{12}$ and in the opposite sense (vector $-f \perp$, ) for $P_{34}$. 


\begin{tabular}{|c|c|c|c|c|c|c|c|c|}
\hline & \multicolumn{4}{|c|}{ Pair $P_{12}$ in first pore } & \multicolumn{4}{|c|}{ Pair $P_{34}$ in second pore } \\
\hline & $\begin{array}{c}\text { shift } \\
(\AA)\end{array}$ & $\begin{array}{l}\text { distance } \\
(\AA)\end{array}$ & $\begin{array}{l}\text { tilt } \\
\left({ }^{\circ}\right)\end{array}$ & $\begin{array}{c}\text { phase } \\
\left(^{\circ}\right)\end{array}$ & $\begin{array}{c}\text { shift } \\
(\AA)\end{array}$ & $\begin{array}{l}\text { distance } \\
(\AA)\end{array}$ & $\begin{array}{l}\text { tilt } \\
\left({ }^{\circ}\right)\end{array}$ & $\begin{array}{c}\text { phase } \\
\left({ }^{\circ}\right)\end{array}$ \\
\hline pX-pX & 3.8 & 3.17 & 23.0 & 246.5 & 2.1 & 3.26 & 24.8 & 21.1 \\
\hline oX-oX & 1.2 & 3.20 & 6.3 & 109.2 & 3.2 & 3.03 & 26.3 & 69.1 \\
\hline$m X-m X$ & 2.8 & 3.11 & 23.3 & 154.3 & 1.3 & 3.26 & 6.5 & 140.3 \\
\hline pX-oX & 1.3 & 3.27 & 5.1 & 216.3 & 1.8 & 3.14 & 5.8 & 219.9 \\
\hline $\mathrm{pX}-\mathrm{mX}$ & 1.8 & 3.57 & 33.3 & 3.0 & 1.7 & 3.24 & 3.1 & -23.0 \\
\hline$o X-m X$ & 2.9 & 3.33 & 34.1 & 56.7 & 1.6 & 3.21 & 9.2 & 49.6 \\
\hline Average & 2.3 & 3.3 & 20.9 & 131.0 & 2.0 & 3.2 & 12.6 & 79.5 \\
\hline
\end{tabular}

Table 2 Geometrical parameters of individual xylene pairs $\mathbf{P}_{12}$ and $\mathbf{P}_{34}$ in their adsorbed state.

Comparison of the geometrical parameters of the adsorbed pairs (Table 2) with the pairs in vacuum (Table 1) shows that the distance is similar to the values for the adsorbed pairs in vacuum and that the shift has increased. Whereas the tilt takes values up to $34.1^{\circ}$ in the adsorbed state, it remains close to zero in vacuum. In gas phase, this could be a consequence of our selection of initial structures where the tilt has been put to zero systematically. When the pair is brought into the pore, the xylenes have to reorient themselves to attain optimal stacking with the terephthalic framework linkers, thus causing the large rotation.

The selectivity of xylene isomers in MIL-47 has earlier been attributed to geometrical packing effects [8, 9]. From X-ray data at high loadings [8, 9], it was suggested that molecules are adsorbed by pairs, with their aromatic rings facing each other. The occurrence of certain geometrical effects for single-component adsorption obtained by Rietveld refinement is as follows: [8]

(1) pX: The methyl groups within a stacked para-xylene pair are perfectly staggered.

(2) oX: Structure refinement of ortho-xylene pairs reveals that the stacking of these isomers is analogous to that of para-xylene, but ring alignment is slightly less effective: the rings are shifted with respect to each other. 
(3) $\mathrm{mX}$ : Within pairs of meta-xylene, a steric interaction arises between an aromatic ring of one molecule and a methyl group of a molecule in the neighboring unit cell in the $a$ direction. This interaction causes a tilt and a rotation of the aromatic molecules, preventing the optimal stacking of the rings.

In the three cases, the methyl groups dictate the spatial arrangement of the pairs. However, these experimental geometries differ from our most stable optimized structures (visualized in Figure 7 and Figures 6-8 of Supp. Info.): the rings of the energetically most stable structures are often not parallel (tilt $\theta$ ) and are shifted (shift $s$ ). Such a more random ordering was also observed by Castillo et al. in GCMC simulations of a fully packed 4x2x2 unit cell [20]. Their unordered embedding of the ortho-xylenes in the pores resembles the configuration of our oX pair $\mathrm{P}_{34}$ [20]. They only found ordering for the $\mathrm{pX}$ pairs, where all $\mathrm{CH}_{3}$ groups between neighboring cells have the same orientation, and which resembles the geometry of our pX pair $\mathrm{P}_{12}$.

\subsection{Decomposition of adsorption energy in MIL-47}

The adsorption energy is influenced by three effects: framework deformation, interaction of xylenes with the pore walls, and stacking of xylenes. The adsorption energy is thus decomposed into three terms

$$
\Delta E_{\text {ads }}=E_{\text {deform }}+E_{\text {inter }}+E_{\text {stack }}
$$

The physical interpretation of the terms is visualized in Figure 8 and the calculated values are reported in Table 3. 


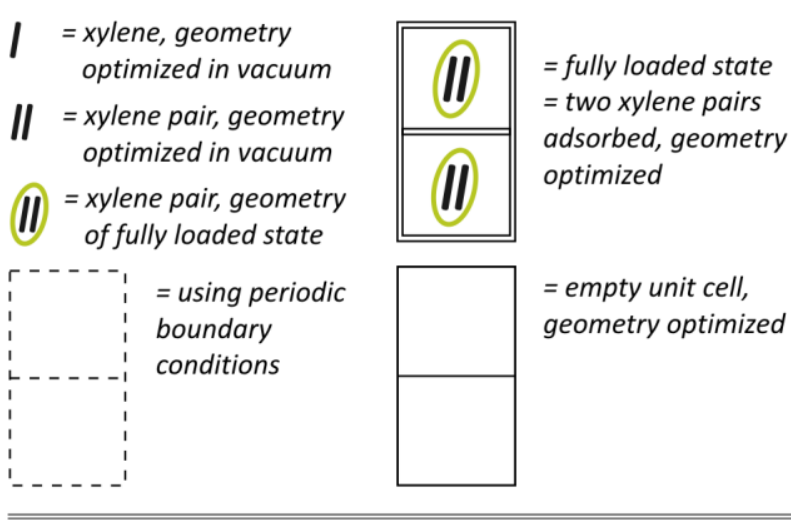

$\Delta E_{a d s}=\frac{\mid \text { II }}{\mid \text { III }}-|-|-\mid$

$E_{\text {deform }}=\square-$

$E_{\text {inter }}=$\begin{tabular}{|l||l|l||}
\hline II \\
\hline \hline II
\end{tabular}

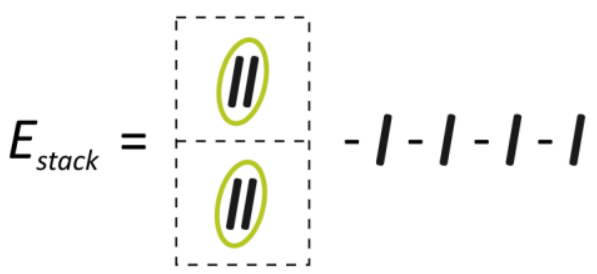

$E_{\text {pair }}\left(P_{12}\right)=\prod-I-I$

$E_{\text {pair }}\left(P_{34}\right)=$ similar

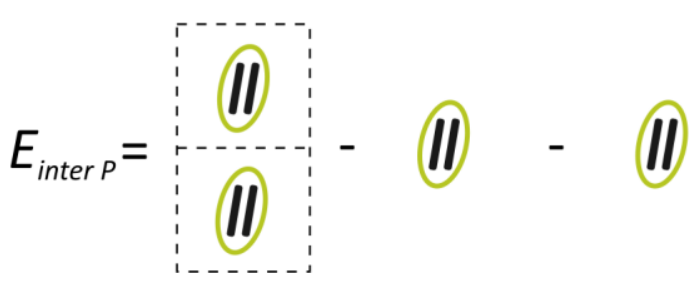

Figure 8 Visualization of the decomposition of the adsorption energy (Eq. (5)): the deformation energy (Eq. (6)), the xylene-framework interaction (Eq. (7)), and the stacking energy (Eq. (10)) are calculated by deleting the xylenes or the framework from the fully loaded framework, and performing static calculations. The stacking energy is further decomposed in pair energies (Eq. (8)) and the inter-pair energy (Eq. (9)). 
A first contribution is the framework deformation. In order to accommodate the xylene pairs in the pores, the structure needs to dilate and the linkers may need to reorient. This effect is quantified by the deformation energy $E_{\text {deform }}$, which is defined as the difference in energy between the empty framework with its geometry as in the fully adsorbed state $(F$,stat) and the empty framework with its geometry relaxed $(F)$,

$$
E_{\text {deform }}=E^{\text {stat }}(F)-E(F)
$$

The framework deformation due to the loading requires energy $\left(E_{\text {deform }}>0\right)$.

A second contribution are the host-guest interactions. The xylenes are physisorbed in the pores due to interactions with the framework. The interaction energy $E_{\text {inter }}$ is defined as the energy difference of the fully loaded framework $\left(F, P_{12}, P_{34}\right)$ with respect to the energy of the empty host $(F$, stat $)$ and the energy of the xylenes pairs $\left(P_{12}, P_{34}\right.$, stat $)$.

$$
E_{\text {inter }}=E\left(F, P_{12}, P_{34}\right)-E^{\text {stat }}(F)-E^{\text {stat }}\left(P_{12}, P_{34}\right)
$$

The latter two static calculations are simple single point energy computations using the same geometry as in the fully loaded host (no geometry optimization is performed) from which the xylenes or the framework are removed (Figure 8). The interaction of the xylenes with the pore walls, in this case mainly the terephthalic linkers, is attractive $\left(E_{\text {inter }}<0\right)$.

The third contribution in Eq. (5) is the interaction $E_{\text {stack }}$ between the xylene molecules. As the xylenes appear in pairs in the pores, a large part of these xylene interactions per unit cell is the stacking energy of the first pair $E_{\text {pair }}\left(P_{12}\right)$ and the stacking energy of the second pair $E_{\text {pair }}\left(P_{34}\right)$. Due to the interaction with the host, the xylene pairs have different geometries than in vacuum. Instead of using Eq. (1), these pair stacking energies should be calculated by comparing the energy of an isolated pair in vacuum with the energies of two individual xylenes in vacuum. For instance, the geometry of the isolated pair $P_{12}$ is obtained by removing the framework and pair $P_{34}$ from the optimized fully loaded state. The static energy of this $P_{12}$ geometry is then calculated with a single point computation in a large box (vacuum, $P_{12}$, stat), 


$$
E_{\text {pair }}\left(P_{12}\right)=E^{\text {stat }, v a c}\left(P_{12}\right)-E^{v a c}\left(X_{1}\right)-E^{v a c}\left(X_{2}\right)
$$

and similarly for pair $P_{34}$. The pair stacking energy is schematically visualized in Figure 8 .

The remainder of the adsorption energy represents interactions between xylene pairs due to the periodicity of the material and is labeled as the inter-pair interaction $E_{\text {interp }}$. A xylene pair interacts with other adsorbed xylene pairs in its own channel as well as with xylene pairs in adjacent channels. The interaction is calculated from the static energy of the pairs in the fully loaded geometry, where the framework has been removed, with the periodicity set to the framework lattice parameters $\left(P_{12}, P_{34}\right.$, stat $)$. This energy is compared with the static energy of the individual pairs, still in the same geometry, using a large box (vacuum, $P_{12}$,stat and vacuum, $P_{34}$, stat).

$$
E_{\text {interP }}=E^{\text {stat }}\left(P_{12}, P_{34}\right)-E^{\text {stat }, v a c}\left(P_{12}\right)-E^{\text {stat }, \text { vac }}\left(P_{34}\right)
$$

This procedure measures the interaction between the two pairs and the interaction with the periodic images of the pairs. Similarly to the stacking energy of two molecules, the inter-pair interaction is favorable for the adsorption $\left(E_{\text {inter } P}<0\right)$. The total stacking energy in Eq. (5), which is also a negative contribution, can now be calculated as the sum of the pair stacking energies and the inter-pair stacking:

$$
E_{\text {stack }}=E_{\text {pair }}\left(P_{12}\right)+E_{\text {pair }}\left(P_{34}\right)+E_{\text {inter } P}
$$

\begin{tabular}{|c|c|c|c|c|c|c|c|c|}
\hline & \multicolumn{3}{|c|}{$\Delta E_{\mathrm{ads}}$} & \multicolumn{3}{|c|}{$E_{\text {stack }}$} & \multirow[b]{2}{*}{$\Delta E_{\text {ads }}$} & \multirow[b]{2}{*}{$\Delta E_{\mathrm{ads}} / 4$} \\
\hline & $E_{\text {deform }}$ & $E_{\text {inter }}$ & $E_{\text {stack }}$ & $E_{\text {pair }}\left(P_{12}\right)$ & $E_{\text {pair }}\left(P_{34}\right)$ & $E_{\text {inter } P}$ & & \\
\hline MIL-47 + 4pX & 19.3 & -120.8 & -131.2 & -29.1 & -18.4 & -83.7 & -232.7 & -58.2 \\
\hline MIL-47 + 4oX & 29.3 & -120.1 & -137.4 & -26.9 & -34.1 & -76.5 & -228.3 & -57.1 \\
\hline MIL-47 + 4mX & 18.8 & -138.3 & -109.2 & -31.8 & -37.2 & -40.3 & -228.8 & -57.2 \\
\hline MIL-47+2pX-oX & 15.6 & -134.4 & -103.9 & -30.9 & -35.9 & -37.2 & -222.8 & -55.7 \\
\hline MIL-47+2pX-mX & 8.0 & -147.0 & -116.0 & -28.8 & -22.3 & -65.0 & -255.0 & -63.8 \\
\hline
\end{tabular}




$\begin{array}{ccccccccc}\text { MIL-47+2oX-mX } & 5.9 & -141.9 & -95.0 & -27.6 & -25.2 & -42.2 & -231.0 & -57.7 \\ \text { Average } & 16.2 & -133.8 & -115.5 & -29.2 & -28.9 & -57.5 & -233.1 & -58.3\end{array}$

Table 3 Energy contributions to the adsorption energy of the fully loaded framework: deformation energy of the framework, interaction energy between xylenes and framework, and xylene stacking energy. The stacking energy consists of the stacking energies of the individual pairs and the inter-pair stacking energy. The adsorption energy per xylene is obtained by division by four. All energies in $\mathrm{kJ} / \mathrm{mol}$.

The predicted total adsorption energies are on average $-233.1 \mathrm{~kJ} / \mathrm{mol}$ per unit cell (Table 3), which amounts to $-58.3 \mathrm{~kJ} / \mathrm{mol}$ per adsorbed xylene molecule. On average, the host-xylene interaction $E_{\text {inter }}$ and the xylene stacking energy $E_{\text {stack }}$ contribute equally. But in individual cases we notice some significant deviations: the total stacking energy can vary by more than $40 \mathrm{~kJ} / \mathrm{mol}$. Nevertheless, this effect is mostly compensated by the interaction energy between the xylenes and the walls of the host. A large stacking energy of the xylenes is accompanied systematically by a higher repulsive deformation energy of the framework. This feature could be understood by assuming that some framework relaxation is required to accommodate the xylene pair in its most favorable stacked conformation.

Within the category of pure pairs, the xylene-framework interaction energy is by far the strongest for the adsorption of pure $\mathrm{mX}$ pairs $(-138.3 \mathrm{~kJ} / \mathrm{mol})$. The trend to favor $\mathrm{mX}$ is maintained when using mixed pairs, since the adsorption of mixed pairs oX-mX or $\mathrm{pX}-\mathrm{mX}$ show by far the largest interaction energies with the host. However, these favorable interactions are partially cancelled out by the lower stacking energies. Note also that more favorable energies for pure pairs not systematically lead to more favorable energies in the mixed pairs. For instance, mixing with the xylene isomer with the strongest stacking energy $(\mathrm{oX})$ does not result in the strongest stacking energies for the mixed pairs (oX-mX, oX-pX). From Table 3 we conclude that the data for pure pairs are insufficient to predict adsorption energetics for mixed pairs.

The decomposition of the stacking energy in Table 3 shows that the pair stacking energies of the adsorbed pairs are less favorable than those of the pairs in vacuum (average -31.7 $\mathrm{kJ} / \mathrm{mol})$. Nevertheless, the pair stacking attains an average stacking efficiency of (-29.228.9) $/ 2=-29.0 \mathrm{~kJ} / \mathrm{mol}$. The inter-pair stacking energy can be very large, up to $-83.7 \mathrm{~kJ} / \mathrm{mol}$ for $\mathrm{pX}$. The variation in the inter-pair stacking is explained by the organization of the xylenes in the pores, which may be derived from the geometrical parameters as follows. It is clear from Figure 6 that $X_{1}$ and $X_{2}$ are nearest neighbors in the channel, and $X_{3}$ and $X_{4}$ are nearest neighbors in the adjacent channel. The interaction of a xylene with its nearest neighbors is 
included in the pair stacking energies $E_{\text {pair }}\left(P_{12}\right)$ and $E_{\text {pair }}\left(P_{34}\right)$. The interaction of xylenes with their next nearest neighbors is then of course included in the $E_{\text {interP }}$. According to our geometry vs. energy analysis in vacuum (Figure 5), the strength of the xylene-xylene interaction is mainly determined by the distance between the two molecules. To see the effect of the distance on the inter-pair energy, we determined the distance from the xylenes to their second nearest neighbor, which should be the dominant contribution. This distance is calculated as the root of $s^{2}+d^{2}$ ( $s$ and $d$ as defined in Figure 2), which equals the distance between the centers of their rings. For pX, the second neighbors are separated by $4.96 \AA$ and $5.77 \AA$, and these relatively short separations result in the strong inter-pair stacking energy of $-83.7 \mathrm{~kJ} / \mathrm{mol}$. In contrast, the second neighbors for $\mathrm{mX}$ are separated by $5.75 \AA$ and $7.40 \AA$, resulting in a much weaker inter-pair energy of $-40.3 \mathrm{~kJ} / \mathrm{mol}$.

The typical stacking energy in vacuum, about $-31.7 \mathrm{~kJ} / \mathrm{mol}$ (Table 1), may be used as a 'unit for energy' to compare the importance of the energy contributions. The interaction energy of two pairs with the framework is worth over four units, the stacking of the pairs is worth one unit each, the inter-pair stacking is worth two units, and the deformation diminishes the total by half a unit. This brings the adsorption energy to nearly eight vacuum stacking energy equivalents $(-233.1 \mathrm{~kJ} / \mathrm{mol})$. The attractive stacking between xylene molecules is here of the same importance as the attractive interaction energy between xylenes and framework.

Summarizing, the pure pairs deform the framework more (larger positive $E_{\text {deform}}$ ) and interact more weakly with the framework (weaker $E_{\text {inter }}$ ) than the mixed pairs. However, the pure xylene pairs stack more efficiently in the pores (stronger $E_{\text {stack }}$ ). Overall, the values of adsorption energy for the various xylene pairs all have the same order of magnitude, and appear not to explain the different adsorption selectivity of the $\mathrm{pX}, \mathrm{oX}$, and $\mathrm{mX}$ isomers. If the pure adsorption isotherms were to be solely determined by the strength of the adsorption energy, one would need a strong adsorption energy for $\mathrm{pX}$, followed by oX, and a considerably weaker adsorption energy for $\mathrm{mX}$. Since this is not the case, our results indicate that the selective adsorption behavior can not be explained purely energetically. This means that temperature and entropy are expected to play a determining role for the selectivity.

The entropy arises from the many possible configurations the xylenes can take when adsorbed in the pores. In general, the more configurations the xylenes can take within the pores, the higher the entropy, and the more favorable the adsorption. A first contribution to the entropy is the relative orientation of the molecules in a xylene pair. The presence of the 
methyl groups can here reduce the number of configurational states. The fewer states are prohibited (because of overlapping methyl groups), the more states are available, and the more entropy is available. A second contribution to the entropy is the positioning of the pair in the channels. Likewise, the pair may take a number of different orientations and positions inside the channels. Bulkier pairs show a more limited number of available configurations, leading to a lower entropy. A third entropic contribution arises in the process of multicomponent adsorption, referred to as mixing entropy. Many ways exist to distribute the xylenes of each kind over the pores. The information about entropy is lacking in adsorption energy calculations, but could be obtained by a vibrational analysis [47, 48], Monte Carlo or Molecular Dynamics simulations. GCMC calculations, as those by Castillo et al. [20], include energetic and entropic effects and agree with experimental single-component isotherms. Our study now shows that the energetics of the pure and mixed xylene adsorption at high loadings are not alone responsible for the selectivities between isomers, such that the separation is also entropically driven. 


\section{Conclusions}

The molecular packing effects inside the confining environment of the MIL-47 pore system have been studied by quantumchemical calculations. The guest-guest interactions are first quantified in the gas phase. Xylenes show a high stacking energy in vacuum, such that xylenes are expected to stack efficiently with the rings of the MIL-47 linkers. When the xylenes are inserted in the MIL-47 pores, not only guest-guest interactions contribute to the adsorption energy, but also host-guest interactions. The adsorption energy for high loadings of para-, ortho-, and meta-xylene has thus been decomposed in three contributions: the deformation energy of the framework, the framework-xylene interaction, and the stacking energy. The stacking energy has been found to be equally important as the framework-xylene interaction, hence confirming the hypothesis that $\pi-\pi$ stacking is responsible for the adsorption. The average stacking energy of xylene pairs in vacuum can be used as a unit for the interactions. The adsorption energy in the fully loaded state amounts to nearly two vacuum stacking equivalents per adsorbed xylene, which explains the efficient adsorption at room temperature.

The energetics could not explain the experimentally observed separation of xylene isomers with a preferential adsorption for orthoxylene and paraxylene, since all adsorption energies lie in the same range. Therefore, entropic effects are likely the main driving force for the adsorption selectivity. This has two implications. First, sampling of configurational space should be adequately performed to assess the entropic effects, which can only be obtained with molecular dynamics or Monte Carlo simulations. Second, since the accuracy of the energetics is of subordinary importance to the sampling, it is expected that reasonable results can be obtained with classical force-fields. Whereas force-fields are less accurate than an ab initio treatment, they allow considerably longer sampling times because of the reduced computational cost.

\section{Acknowledgements}

This work was supported by the Fund for Scientific Research - Flanders (FWO), the research Board of Ghent University, and BELSPO in the frame of IAP 6/27. Funding was also received from the European Research Council under FP7 with ERC grant agreement number 240483. A.G., T.V., and M.A.vdV. are post-doctoral researchers of the Fund for Scientific Research - Flanders (FWO). The computational resources (Stevin Supercomputer 
Infrastructure) and services used in this work were provided by Ghent University, the Hercules Foundation and the Flemish Government - department EWI.

Keywords: Adsorption • MIL-47 • DFT-D • xylenes $\bullet$ separation

\section{References}

[1] G. Ferey, Hybrid porous solids: past, present, future, Chem. Soc. Rev., 37 (2008) 191-214.

[2] J.R. Long, O.M. Yaghi, The pervasive chemistry of metal-organic frameworks, Chem. Soc. Rev., 38 (2009) 1213-1214.

[3] J.J. Perry, J.A. Perman, M.J. Zaworotko, Design and synthesis of metal-organic frameworks using metal-organic polyhedra as supermolecular building blocks, Chem. Soc. Rev., 38 (2009) 1400-1417.

[4] M. Eddaoudi, J. Kim, N. Rosi, D. Vodak, J. Wachter, M. O'Keeffe, O.M. Yaghi, Systematic design of pore size and functionality in isoreticular MOFs and their application in methane storage, Science, 295 (2002) 469-472.

[5] G. Ferey, C. Serre, T. Devic, G. Maurin, H. Jobic, P.L. Llewellyn, G. De Weireld, A. Vimont, M. Daturi, J.S. Chang, Why hybrid porous solids capture greenhouse gases?, Chem. Soc. Rev., 40 (2011) 550-562.

[6] S.T. Meek, J.A. Greathouse, M.D. Allendorf, Metal-Organic Frameworks: A Rapidly Growing Class of Versatile Nanoporous Materials, Adv. Mater., 23 (2011) 249-267.

[7] D.J. Tranchemontagne, J.L. Mendoza-Cortes, M. O'Keeffe, O.M. Yaghi, Secondary building units, nets and bonding in the chemistry of metal-organic frameworks, Chem. Soc. Rev., 38 (2009) $1257-$ 1283.

[8] L. Alaerts, C.E.A. Kirschhock, M. Maes, M.A. van der Veen, V. Finsy, A. Depla, J.A. Martens, G.V. Baron, P.A. Jacobs, J.E.M. Denayer, D.E. De Vos, Selective adsorption and separation of xylene isomers and ethylbenzene with the microporous vanadium(IV) terephthalate MIL-47, Angew. Chem.Int. Edit., 46 (2007) 4293-4297.

[9] V. Finsy, H. Verelst, L. Alaerts, D. De Vos, P.A. Jacobs, G.V. Baron, J.F.M. Denayer, Pore-fillingdependent selectivity effects in the vapor-phase separation of xylene isomers on the metal-organic framework MIL-47, Journal of the American Chemical Society, 130 (2008) 7110-7118.

[10] F. Vermoortele, M. Maes, P.Z. Moghadam, M.J. Lennox, F. Ragon, M. Boulhout, S. Biswas, K.G.M. Laurier, I. Beurroies, R. Denoyel, M. Roeffaers, N. Stock, T. Düren, C. Serre, D.E. De Vos, pXylene-Selective Metal-Organic Frameworks: A Case of Topology-Directed Selectivity, Journal of the American Chemical Society, 133 (2011) 18526-18529.

[11] Ullmann's Encyclopedia of Industrial Chemistry, 6th ed., in, electronic release, 2000.

[12] K. Barthelet, J. Marrot, D. Riou, G. Ferey, A breathing hybrid organic-inorganic solid with very large pores and high magnetic characteristics, Angew. Chem.-Int. Edit., 41 (2002) 281-+.

[13] S. Grimme, Semiempirical GGA-type density functional constructed with a long-range dispersion correction, J. Comput. Chem., 27 (2006) 1787-1799.

[14] S. Grimme, J. Antony, T. Schwabe, C. Muck-Lichtenfeld, Density functional theory with dispersion corrections for supramolecular structures, aggregates, and complexes of (bio)organic molecules, Org. Biomol. Chem., 5 (2007) 741-758.

[15] T. Schwabe, S. Grimme, Theoretical thermodynamics for large molecules: Walking the thin line between accuracy and computational cost, Accounts Chem. Res., 41 (2008) 569-579.

[16] B. Liu, B. Smit, Comparative Molecular Simulation Study of CO2/N2 and CH4/N2 Separation in Zeolites and Metal\&\#x2212;Organic Frameworks, Langmuir, 25 (2009) 5918-5926.

[17] N.A. Ramsahye, G. Maurin, S. Bourrelly, P.L. Llewellyn, T. Devic, C. Serre, T. Loiseau, G.

Ferey, Adsorption of $\mathrm{CO} 2$ in metal organic frameworks of different metal centres: Grand Canonical Monte Carlo simulations compared to experiments, Adsorpt.-J. Int. Adsorpt. Soc., 13 (2007) 461-467.

[18] N. Rosenbach, H. Jobic, A. Ghoufi, F. Salles, G. Maurin, S. Bourrelly, P.L. Llewellyn, T. Devic, C. Serre, G. Ferey, Quasi-elastic neutron scattering and molecular dynamics study of methane 
diffusion in metal organic frameworks MIL-47(V) and MIL-53(Cr), Angew. Chem.-Int. Edit., 47 (2008) 6611-6615.

[19] F. Salles, H. Jobic, G. Maurin, M.M. Koza, P.L. Llewellyn, T. Devic, C. Serre, G. Ferey, Experimental evidence supported by simulations of a very high H-2 diffusion in metal organic framework materials, Phys. Rev. Lett., 100 (2008).

[20] J.M. Castillo, T.J.H. Vlugt, S. Calero, Molecular Simulation Study on the Separation of Xylene Isomers in MIL-47 Metal-Organic Frameworks, The Journal of Physical Chemistry C, 113 (2009) 20869-20874.

[21] S. Wang, Q. Yang, C. Zhong, Adsorption and separation of binary mixtures in a metal-organic framework Cu-BTC: A computational study, Separation and Purification Technology, 60 (2008) 30-35. [22] L. Hamon, P.L. Llewellyn, T. Devic, A. Ghoufi, G. Clet, V. Guillerm, G.D. Pirngruber, G. Maurin, C. Serre, G. Driver, W.v. Beek, E. Jolimaître, A. Vimont, M. Daturi, G.r. Férey, Coadsorption and Separation of CO2-CH4 Mixtures in the Highly Flexible MIL-53(Cr) MOF, Journal of the American Chemical Society, 131 (2009) 17490-17499.

[23] M. Gallo, D. Glossman-Mitnik, Fuel Gas Storage and Separations by Metal\&\#x2212; Organic Frameworks: Simulated Adsorption Isotherms for $\mathrm{H} 2$ and $\mathrm{CH} 4$ and Their Equimolar Mixture, The Journal of Physical Chemistry C, 113 (2009) 6634-6642.

[24] L. Pan, D.H. Olson, L.R. Ciemnolonski, R. Heddy, J. Li, Separation of hydrocarbons with a microporous metal-organic framework, Angew. Chem.-Int. Edit., 45 (2006) 616-619.

[25] L. Vanduyfhuys, T. Verstraelen, M. Vandichel, M. Waroquier, V. Van Speybroeck, Ab initio parametrized force field of the metal-organic framework MIL-53(Al) for use in molecular simulations including lattice dynamics, in preparation (2012).

[26] M.O. Sinnokrot, C.D. Sherrill, Unexpected Substituent Effects in Face-to-Face $\pi$-Stacking Interactions, The Journal of Physical Chemistry A, 107 (2003) 8377-8379.

[27] M.O. Sinnokrot, C.D. Sherrill, Highly accurate coupled cluster potential energy curves for the benzene dimer: Sandwich, T-shaped, and parallel-displaced configurations, J. Phys. Chem. A, 108 (2004) 10200-10207.

[28] M.O. Sinnokrot, C.D. Sherrill, Substituent Effects in $\pi-\pi$ Interactions: Sandwich and T-Shaped Configurations, Journal of the American Chemical Society, 126 (2004) 7690-7697.

[29] L. Alaerts, M. Maes, P.A. Jacobs, J.F.M. Denayer, D.E. De Vos, Activation of the metal-organic framework MIL-47 for selective adsorption of xylenes and other difunctionalized aromatics, Phys. Chem. Chem. Phys., 10 (2008) 2979-2985.

[30] D. Cremer, J.A. Pople, General definition of ring puckering coordinates, Journal of the American Chemical Society, 97 (1975) 1354-1358.

[31] R. Car, M. Parrinello, Unified Approach for Molecular Dynamics and Density-Functional Theory, Phys. Rev. Lett., 55 (1985) 2471-2474.

[32] A.D. Becke, Density-functional exchange-energy approximation with correct asymptotic behavior, Physical Review A, 38 (1988) 3098.

[33] D. Vanderbilt, Soft self-consistent pseudopotentials in a generalized eigenvalue formalism, Physical Review B, 41 (1990) 7892.

[34] T. Verstraelen, V. Van Speybroeck, M. Waroquier, ZEOBUILDER: A GUI toolkit for the construction of complex molecular structures on the nanoscale with building blocks, J. Chem Inf. Model., 48 (2008) 1530-1541.

[35] J. Gu, J. Wang, J. Leszczynski, Y. Xie, H.F. Schaefer, Chem. Phys. Lett., 459 (2008) 164.

[36] G.B. McGaughey, M. Gagne, A.K. Rappe, J. Biol. Chem., 273 (1998) 15458.

[37] C. Morgado, M.A. Vincent, I.H. Hillier, X. Shan, Phys. Chem. Chem. Phys., 9 (2007) 448.

[38] M. Pavone, N. Rega, V. Barone, Chem. Phys. Lett., 452 (2008) 333.

[39] M. Piacenza, S. Grimme, J. Comput. Chem., 25 (2004) 83.

[40] R. Podeszwa, R. Bukowski, K. Szalewicz, J. Phys. Chem. A, 110 (2006) 10345.

[41] M.P. Waller, A. Robertazzi, J.A. Platts, D.E. Hibbs, P.A. Williams, J. Comput. Chem., 27 (2006) 491.

[42] Y. Zhao, D.G. Truhlar, Phys. Chem. Chem. Phys., 7 (2005) 2701.

[43] Y. Zhao, D.G. Truhlar, J. Chem. Theory Comput., 2 (2006) 1009. 
[44] E.C. Lee, B.H. Hong, J.Y. Lee, J.C. Kim, D. Kim, Y. Kim, P. Tarakeshwar, K.S. Kim, Substituent effects on the edge-to-face aromatic interactions, Journal of the American Chemical Society, 127 (2005) 4530-4537.

[45] R.K. Raju, J.W.G. Bloom, Y. An, S.E. Wheeler, Substituent Effects on Non-Covalent Interactions with Aromatic Rings: Insights from Computational Chemistry, ChemPhysChem, 12 (2011) 3116-3130. [46] E.G. Hohenstein, J.N. Duan, C.D. Sherrill, Origin of the Surprising Enhancement of Electrostatic Energies by Electron-Donating Substituents in Substituted Sandwich Benzene Dimers, Journal of the American Chemical Society, 133 (2011) 13244-13247.

[47] B.A. De Moor, A. Ghysels, M.-F.o. Reyniers, V. Van Speybroeck, M. Waroquier, G.B. Marin, Normal Mode Analysis in Zeolites: Toward an Efficient Calculation of Adsorption Entropies, Journal of Chemical Theory and Computation, 7 (2011) 1090-1101.

[48] J. Van der Mynsbrugge, K. Hemelsoet, M. Vandichel, M. Waroquier, V. Van Speybroeck, An Efficient Approach for the Computational Study of Alcohol and Nitrile Adsorption in H-ZSM-5, The Journal of Physical Chemistry C, (2012). 